\title{
Die Narrative der digitalen Transformation
}

\section{Der unaufhaltsame Transfer von Dingen, Handlungen und Bedürfnissen in Daten - Worauf sollten wir uns einstellen? Welche Gestaltungsräume bleiben Deutschland und der EU für ein eigenständiges Narrativ?}

\author{
Arno Rolf ${ }^{1}$ \\ Online publiziert: 18 . September 2020 \\ ๑) Springer-Verlag GmbH Deutschland, ein Teil von Springer Nature 2020
}

\section{Zusammenfassung}

Worauf muss sich die europäische Gesellschaft in der digitalen Transformation einstellen? Welche Gestaltungsräume verbleiben für die Europäische Union angesichts der beiden dominierenden Wettbewerber China und USA? Ihre Narrative werden analysiert. Es wird ein Digitalisierungspfad für die EU unter Berücksichtigung von unvorhersehbaren Ereignissen sowie nutzbarer Regulierungs- und Innovationspotenziale entworfen. Es wird ein Narrativ entwickelt, das auf die Wiedergewinnung der verlorengegangenen europäischen Regulierungshoheit, die Stärken der Ökonomie und eine nachhaltige Pfadentwicklung setzt.

Worauf läuft der Pfad der digitalen Transformation mit seinen Bausteinen und Herausforderungen hinaus? Was steht am Ende der Erzählungen? Mit welchen Metaphern werden sie uns nahegebracht? Worauf sollten wir uns einstellen?

Mit der digitalen Transformation verweben sich politische und private Machtinteressen, Deutungskämpfe, Narrative und Metaphern zu einem Digitalisierungspfad. Der Pfad macht Kapitalinteressen, Konflikte, Sieger und Verlierer sichtbar. Die vermeintliche „Nutzungslogik“ stellt sich als eine Erzählung bzw. ein Narrativ heraus, Kämpfe werden ausgetragen, Interessen und Werte sich durchsetzen.

Mit diesen Themen setzen sich traditionell die Sozialwissenschaften auseinander. Wünschenswert wäre, dass ihre Erkenntnisse in die Wirtschafts- und Ingenieurwissenschaften und auch in die Informatik einfließen würden. Über die Veränderung wissenschaftlicher Narrative forscht seit Langem die Wissenschaftstheorie, erwähnt seien unter vielen Thomas Kuhns Arbeiten zum Paradigmenwechsel [1] und Ludwig Flecks frühe Überlegungen zur Entstehung und Entwicklung wissenschaftlicher Tatsachen [2].

Im Folgenden wird versucht, aus informationstechnischen Potenzialen, Bausteinen, Herausforderungen, domi-

Arno Rolf

rolf@informatik.uni-hamburg.de

1 Fachbereich Informatik, Universität Hamburg, Hamburg, Deutschland nierenden Erzählungen und Metaphern ein Mosaik zusammenzufügen, das Auskunft geben kann, worauf sich die europäische Gesellschaft und Ökonomie angesichts der digitalen Transformation einstellen sollten und welche Gestaltungsräume verbleiben. Welche Möglichkeiten hat die EU, ein eigenständiges Narrativ zu entwickeln?

\section{Die Verwandlung der Gesellschaft durch Entstofflichung}

Einige bedeutende wissenschaftliche Arbeiten lassen sich unter die Begriffe Transformation oder Disruption subsumieren, etwa Josef L. Schumpeters ,schöpferische Zerstörung“. Der Begriff Transformation taucht 1944 im Titel des Buches von Karl Polanyi „The Great Transformation“ auf [3]. Polanyi beschreibt die Entstehung einer unregulierten liberalen Marktwirtschaft, die die Ökonomie aus gesellschaftlichen Zusammenhängen des Gemeinwohls herauslöste, was am Ende zu Krieg führte.

Zurück zur digitalen Transformation. Ein Kern der digitalen Transformation ist die Entstofflichung vieler Dinge und die unaufhaltsame Verwandlung von Handlungen und Bedürfnissen in Daten.

Einige Beispiele: Viele Informationen erreichen uns heute über Mails, Apps, Messengerdienste; Bücher, Zeitungen, Magazine verlieren nach und nach ihren materiellen Cha- 
Neue Bedürfnisse

durch "rein digitale Produkte":

Facebook, Suchmaschine...

Zerstörung materieller Dinge durch Entstofflichung: Buch-

handel, Filmproduktion, Fotografie...

Digitalisierung von Alltags-

handlungen: bargeldlose

Zahlungen, Tickets, Bordkarten...

Digitalisierung von Dienstleistungen: Einzelhandel/Amazon, $>$ Dating-Agenturen; Taxis/Uber...

Konstruktion physischer Dinge ohne Arbeit (KI) durch: Industrieroboter...

"Hybride Produkte": Anreicherung physischer Dinge mit Intelligenz: Maschinen, Autos...

\section{$>$}

\section{$>$}


$>$ f

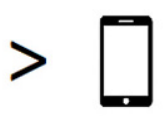

.
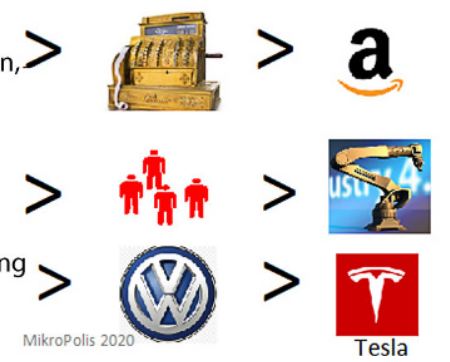

Abb. 1 Beispiele der Verwandlung von Produkten, Bedürfnissen und Dienstleistung in Daten

rakter; Kauf und Speicherung von Tickets werden häufig über unser Smartphone abgewickelt; es ist nicht mehr unüblich, einen Partner über Dating-Agenturen zu suchen; traditionelle Produkte wie Maschinen und Autos verändern sich durch Daten und künstliche Intelligenz (KI), sie werden zu hybriden Produkten, etwa beim autonom fahrenden Auto; durch Digitalisierung sind auch neue attraktive Bedürfnisse entstanden, die schnelle Verbreitung gefunden haben, u.a. Twitter oder Googles Suchmaschine (Abb. 1).

Durch den Transfer von Produkten, Handlungen und Bedürfnissen in Daten konnte sich die globale Ökonomie in eine Plattformökonomie verwandeln [4]. Auch Netzwerkeffekte haben zu deren rasantem Wachstum beigetragen. Ein digitaler Anbieter, der gleich zu Beginn durch ein innovatives Angebot viele Interessenten auf sich ziehen kann, wird schnell wachsen. Je mehr gleich am Anfang dabei sind, umso mehr kommen hinzu, die mit ihren Informationen und Eingaben die Datenbank füllen, was wiederum die Attraktivität des Anbieters und seines Datenschatzes steigert. Der Erfolg füttert sich selbst.

Die Bürger der westlichen Hemisphäre haben heute akzeptiert, dass vorwiegend amerikanische Plattformen der Big Five Amazon, Apple, Facebook, Google und Microsoft sich in die traditionellen globalen ökonomischen Wertschöpfungsprozesse quetschten und dass diese die digitale Transformation mit ihren Plattformen kontrollieren. Um ihre ursprünglichen Kernprodukte haben sie eine Vielzahl von zusätzlichen Innovationen entwickelt; sie haben Startups aufgekauft und Allianzen mit Weltmarktkonzernen der Old Economy abgeschlossen und stellen am Ende selbst diese in den Schatten, sodass manche von ihnen abhängig wurden.
China, USA, Europa - Analyse ihrer Narrative

Angesichts ihrer Unterschiede in Kultur, Politik und Ökonomie sollten in den drei Blöcken China, USA und Europa unterschiedliche Narrative der digitalen Transformation erkennbar sein.

\section{China}

In China dominieren die drei Datenmonopole Alibaba, Tencent und Baidu, die im engen Austausch mit staatlichen Stellen stehen. Das Land will bereits in diesem Jahr die „harmonische Gesellschaft“ mit dem sogenannten „Sozialkreditsystem“ realisieren: mit Gesichtserkennungssoftware zusammen mit Belohnungs- und Diskrimierungssystemen soll der einzelne Bürger kontrolliert und gesteuert werden.

Chinas Mission ist, den autoritären Staatskapitalismus in Kombination mit Marktwirtschaft und den Möglichkeiten von Big Data und KI auf ein neues Level zu heben. Einerseits will man so die volkswirtschaftliche Produktivität erhöhen und die herrschende Korruption einhegen. Mit dem umfassenden Einsatz der Mustererkennungswerkzeuge Big Data und KI sollen andererseits demokratische Regungen der Bürger in Schach gehalten werden.

Die Parteiführung verspricht sich auf diese Weise, die Verhaltensweisen der Menschen, die Ökonomie wie die gesamte Infrastruktur des Staates für sich transparent und regulierbar machen zu können. Mit den gespeicherten Datenmassen und Mustererkennungssystemen soll das gesellschaftliche Geschehen mit seinen verborgenen Verknüpfungen, Wechsel- und Nebenwirkungen, die bislang jenseits menschlicher Wahrnehmungsmöglichkeiten lagen, erkannt werden. Verhaltensweisen jedes einzelnen, aber auch die Produktivkräfte der Ökonomie können dann, so die Hoffnung, auf verborgene, für die Parteiführung relevante Muster identifiziert, überprüft und in die gewünschte Richtung gelenkt werden (Predictive Analytics); einfacher: Die Erziehung und Manipulation der Massen, der Kultur wie die Steigerung des Wirtschaftswachstums soll so gelingen. Die Staatsführung möchte individuelle und gesellschaftlich unerwünschte Diskussionen durch Mustererkennung erkennen und verhindern.

\section{USA}

Die USA können sich, was die Big-Data- und KI-Potenziale betrifft, mit China in Qualität und Ausmaß messen. Man kann darüber spekulieren, ob China die Vereinigten Staaten bei der digitalen Schlüsseltechnologie KI schon überflügelt hat. KI und Big Data lassen sich in China wahrscheinlich besser perfektionieren, weil dort liberale Bedenken gegen die Ausbeutung der Privatsphäre fehlen, sodass persönliche 
Daten ohne große Probleme in Algorithmen fließen können [5].

Die Datenherrschaft liegt in den demokratisch verfassten Vereinigten Staaten bei den fünf Datenmonopolen Google, Amazon, Facebook, Apple und Microsoft sowie bei den in ihren Schatten wachsenden Plattformen Airbnb, Uber, Netflix u. a.m. Der US-Sicherheitsapparat in Form der NSA kann über den Patriot Act auf diese Daten zugreifen, wovon er auch wohl reichlich Gebrauch macht [6].

Die Frage liegt nahe, ob die US-Internetkonzerne nicht schon lange dabei sind, ein den chinesischen Konkurrenten ähnliches Narrativ zu realisieren, nur auf die monopolkapitalistische Art, das weit weniger in der öffentlichen Diskussion negativ belegt ist als die chinesische Variante? Facebooks Versuch, die private Weltwährung Libra zu etablieren, ist ein besonders dreister Versuch, der in diese Richtung geht. In der Mustererkennung gehören die Big Five zu den globalen Vorreitern bei der Entwicklung wie der Anwendung der „Datenscheunentore“ mit Predictive Anaytics und Profiling. ,Als quasi hoheitliche Akteure kontrollieren sie die zentralen Zugänge zum Internet, strukturieren und observieren die Bewegungsmöglichkeiten der Nutzer, kuratieren und korrigieren im großen Stil Inhalte, Informationsflüsse und Diskussionen“, so Ulrich Dolata [7].

Dolata nennt das „Regulierung durch Plattformen“ und definiert zwei Regelungsbereiche, die die US-Plattformen okkupiert haben: zum einen die privatwirtschaftliche Organisierung und Regulierung der Märkte, auf denen die Plattformen die Koordination der Marktprozesse übernommen haben und die Wettbewerbsbedingungen bestimmen. Sie haben den staatlichen Institutionen das Heft aus der Hand genommen. Zum anderen die Regulierung des Nutzerverhaltens, wo sie über alle nationalen Grenzen hinweg nicht nur ihre Spuren hinterlassen, sondern unabänderliche Tatsachen geschaffen haben [7].

Der ehemalige Bundesverfassungsrichter Wolfgang Hoffmann-Riem weist zu Recht darauf hin, dass sich die von Dolata sogenannte privatwirtschaftliche Landnahme des Internets weitgehend unbemerkt für die Nutzer vollzogen habe, und zwar vorwiegend durch Einbettung von Regulierungen in Software und Standardeinstellungen. Die Politik lasse das bisher geschehen, sie übertrage Google \& Co. damit ihre eigentlich nicht übertragbare Regelungsverantwortung [8]. In ähnlicher Weise hat dies bereits 1999 Lawrence Lessig mit seiner auch heute noch oft zitierten Metapher „Code is law“ ausgedrückt [9].

In den USA gibt es aber eben auch eine kritische liberale Öffentlichkeit - Beispiel Soshana Zuboff -, die auf die denkbare Zerstörung der Demokratie hinweist und die Einhaltung von Werten und Normen der Verfassung einfordert, allerdings mit mäßigem Erfolg [10]. Beide Narrative sind also in den USA vorhanden.

\section{Die Europäische Union (EU)}

Die EU ist eingeklemmt im Westen von globalen Plattformen aus dem Silicon Valley und im Osten von den digitalen Herrschaftsansprüchen Chinas, die über Strategien wie die Seidenstraße immer deutlicher werden. Beide Mächte gründen ihre Herrschaft auf informationstechnischem Vorsprung und riesigen Datenschätzen. Beide haben das System der Datenanalyse und des Profilings ihrer Bürger bzw. Konsumenten perfektioniert. China, um seine kommunistische Staatsführung stabil zu halten und Wachstum zu generieren, die USA zur Profitmehrung und Stabilisierung weniger globaler Plattformen, bei Zugriffsfreiräumen für die NSA.

Chinas Weg ist mit den Ansprüchen einer digitalen sozialen europäischen Gesellschaft unvereinbar, auch wenn vorstellbar ist, dass Staaten wie Ungarn, Polen oder Rumänien eine stille Sympathie für das chinesische Modell haben könnten. Die USA haben bei der digitalen Transformation nicht nur einen technischen Vorsprung gegenüber Europa, sondern regulieren mit ihren privaten Plattformen unsere Märkte und das Verhalten unserer Nutzer. Sie beherrschen mit ihren Plattformen einen immer wichtiger werdenden Teil der Ökonomie und fordern damit unser Wertesystem permanent und grundlegend heraus.

\section{Anpassungszwänge für die EU?}

Soviel zur Beschreibung der digitalen Gegenwart. Es bleibt die Eingangsfrage, worauf wir uns am Ende der Erzählungen einstellen sollten und welche Möglichkeiten der EU bleiben, ein eigenständiges Narrativ zu entwickeln.

Wenn es um die Beschreibung der zu erwartenden digitalen Zukunft geht, geben viele Autorinnen und Autoren nur allgemeine Einschätzungen ab, die sich anlehnen an die Erzählungen, die wir von den Big Five her kennen. So etwa die Industriesoziologin Sabine Pfeiffer, die von der Etablierung eines globalen „Öko-Systems“ ausgeht, das alle globalen Stoff- und Geldströme einbezieht, bei gleichzeitigem Datenzugriff auf den Menschen in seinen Rollen als Bürger, Beschäftigter und Konsument. Das System wie die Menschen würden dann in ihren unterschiedlichen Rollen steuer- und kontrollierbar sein [11].

Ähnlich die Erzählung von Klaus Schwab, Präsident des Davos Forums: ,Alles wird in Form von Daten ausgedrückt, was zu einer Wirtschaft mit unbegrenzter Mobilität führt. Daten werden in Plattformen integriert, wodurch wirtschaftliche Interaktionen bestimmte Vermittler der Vergangenheit nicht mehr brauchen. Die künstliche Intelligenz verwandelt Datenplattformen in intelligente Systeme, die alle Aspekte des Lebens betreffen. Plattformen und Systeme migrieren in die Cloud. Und: Die Systeme bewegen sich von der rei- 
nen Analytik hin zu Vorhersage und daraus abgeleiteten Vorschriften“ [12].

Es ist nicht unwahrscheinlich, dass es in Teilen so kommen wird, wie Sabine Pfeiffer und Klaus Schwab das beschreiben. Sie gehen davon aus, dass die Globalisierung in der chinesischen wie in der US-Variante fortgeschrieben werden muss. Sie gehen allerdings nicht auf den dahin führenden Digitalisierungspfad ein, der mit Konflikten und Kämpfen um Narrative und Metaphern gespickt ist und der Unsicherheit, dass stabil erscheinende gesellschaftliche Abläufe auch kippen können.

\section{Gestaltungsräume der EU durch Ereignisse, Regulierungen und Innovationen}

\section{Ereignisse}

Der Digitalisierungspfad kann anders verlaufen, sofern Ereignisse bedeutsam werden. Etwa die wachsende Deglobalisierung auch aufgrund der COVID-19-Pandemie. HIV, Schweingrippe, Ebola zeigen, dass sich Pandemien in einer globalisierten Welt nicht eingrenzen lassen. Die COVID19-Pandemie bewirkte quasi über Nacht und massenhaft eine Veränderung der Arbeitsorganisation hin zum Homeoffice. Die Entstofflichung der Ökonomie wurde so durch Nutzung von Videokonferenzsystemen durch Entkörperlichung der sozialen Beziehungen ergänzt.

Ereignisse werfen am Beispiel von COVID-19 zahlreiche Frage auf: Werden die großen Internetkonzerne einen digitalen Wachstumsschub zulasten der vielen kleinen Betriebe durchsetzen? Wird das Abreißen globaler Lieferketten durch den Trend zur Deglobalisierung zu stärkerer Regionalisierung innerhalb der EU führen und die Macht der globalen Plattformen reduzieren? Welche Veränderungen wird die Dekabonisierung vor dem Hintergrund einer stringenten Umwelt- und Klimapolitik nach sich ziehen? Was verändert das denkbare Anschwellen der Flüchtlingsströme? Welche Folgen würde die Beendigung unseres Wachstumsmodells mit Externalisierung der Umweltkosten hin zur Kreislaufwirtschaft haben? Was, wenn immer mehr Bürger zur Einsicht gelangen, die Welt sei auch durch Digitalisierung überdreht und bedürfe der Entschleunigung?

Ereignisse wie etwa COVID-19 vermag digitalen Euphorikern wie dem Silicon-Valley-Investor Balaji Srimvasian Aufmerksamkeit zu geben: „Rausziehen in ländliche Gebiete; von zu Hause aus arbeiten; Jobs leichter wechseln in einen wahrhaft globalen Arbeitsmarkt; weniger, aber längere Autofahrten mit autonomen Autos; Lieferdrohnen rund um die Uhr; sozialer Umgang online und in der virtuellen Realität“ [13].

In der COVID-19-Situation ist es wohl normal, Epochenbrüche für möglich zu halten und sie in hellsten oder dunkelsten Farben auszumalen. Mehr überzeugt die Interpretation von Andreas Reckwitz: Für ihn bahnt sich seit Längerem durch Postindustrialisierung, Globalisierung und Digitalisierung ein Politikwechsel an, der durch die Corona-Krise zu einem Paradigmenwechsel ermutigt wird, mit der Folge, dass bislang ökonomisch hochdynamisierte Staaten, ,nicht noch weiter mobilisiert und dereguliert, sondern stabilisiert und reguliert (werden). Eines Staates, der nicht mitläuft, sondern gegenhält, der nicht beschleunigt, sondern eindämmt" [14]. Gefragt ist der Infrastrukturstaat, der die Qualität öffentlicher Güter und Einrichtungen sichert, sowie der resiliente Staat, der für Gefährdungen wie Pandemien, Crashs oder Klimawandel durch Regulierungen vorsorgt.

\section{Regulierungen}

Es ist nicht ratsam, sich auf solche Ereignisse zu verlassen, sondern bei der scheinbaren „Nutzungslogik“ des Digitalisierungspfades anzusetzen. Er wird „gemacht durch Macht“, durch Regulierungen und dadurch, dass Werte beim Design in die Softwaresysteme einfließen [15]. Er verläuft heute beginnend mit Entstofflichung, über die Datenscheunentore der Plattformen von Google, Facebook \& Co. mit ihrem Profiling und ihrem scheinbar niedlichen „Anstupsen“ der Nutzer, was man besser als Manipulation bezeichnet.

Um dies zu verändern, braucht es mutige Regulierungen der nationalen wie EU-Institutionen, die über die Datenschutzgrundverordnung hinausgehen. Die DSGV verhindert eben nicht das Tracking und die Auswertung privater Handlungen und Bedürfnissen von Individuen. Die Datenscheunentore sind weiter weit geöffnet, nicht zuletzt weil der Plattformzutritt durch die Nutzer nur durch ihre Einwilligung in großzügige Datenerhebungen und Verwertungen möglich ist. Solange dies so ist, bleibt der von Soshana Zuboff erwähnte Verhaltensüberschuss eine stabile Bank für profitträchtige Geschäftsmodelle. Und der Nutzer muss die Ausbeutung und Steuerung seiner Einzigartigkeit durch Internetkonzerne akzeptieren.

Ein Instrument wäre die e-Privacy-Verordnung, die bereits vom europäischen Parlament verabschiedet wurde, aber von der dritten Instanz, dem EU-Rat, blockiert wird. Durch die e-Privacy-Verordnung soll die Stärkung der Privatsphäre erreicht werden, indem Vorgaben zum Nutzertracking vor allem durch Cookies gemacht werden, die so u. a. das Erstellen von Nutzerprofilen durch websiteübergreifende Datensammlungen verhindern sollen. Cookies dienen primär zur Identifikation der Webseitenbesucher oder enthalten Informationen über die Aktivitäten des Nutzers auf den Webseiten. Der EuGH hat ebenfalls kürzlich in einem Urteil das Tracking generell nur nach expliziter Zustimmung durch die Anwender nach dem Opt-in-Prinzip erlaubt. Mit der Nichtverabschiedung der e-Privacy- 
Verordnung würde die Chance vergeben, einen einheitlichen Rechtsrahmen zu schaffen, an den sich alle in der EU mit Telekommunikationsleistungen befassten Unternehmen halten müssen, also auch die hier tätigen USInternetkonzerne.

Eine weitere konkrete politische Option sollte die Regulierung der Märkte und Wettbewerbsbedingungen sein, die, wie von Dolata beschrieben, von den Internetkonzernen okkupiert wurden. Neben dem Kartellrecht bliebe als wirklich scharfes Schwert, was aber nur einige mutige Wissenschaftler auspacken, die Entflechtung, Ausgliederung bzw. Zerschlagung der Internetkonzerne beispielsweise des Ökosystems Facebook, WhatsApp, Instragram oder von Google und YouTube, was in den USA diskutiert wird und wo Google möglicherweise ein Verfahren zu erwarten hat.

Möglicherweise gibt es für die EU aber auch subtile Waffen, die die ökonomische Macht der Internetkonzerne einschränken können. Das Stichwort ist Identitätsmanagement. In der physischen Welt gibt es mit dem Personalausweis seit Langem einen Generalschlüssel, der die Identität nachweist. Die digitale Transformation macht einen digitalen Identitätsnachweis notwendig. In Deutschland und einigen EU-Staaten wurden bereits erste Lösungen mit einem universell nutzbaren Login umgesetzt, dem sogenannten Single Sign On. Der Nutzer muss sich nur einmal bei einer entsprechenden Identitätsplattform registrieren. Mit dem dort angelegten Konto besitzt er eine Authentifizierungslegitimation für alle dort assoziierten Unternehmen.

Fehlende nationale Identitätslösungen sind ein unüberwindbares Hindernis für die Etablierung der europäischen digitalen Souveränität, die bislang von den US-Plattformen bestimmt werden. Umgekehrt wäre es ein Pfund für die EU, da die Big Five bislang noch das Defizit haben, deregulierte Identitätsplattformen mit nicht verifizierten Identitäten zu sein. Erste Forschungsergebnisse zeigen, dass Initiativen zum Identitätsmanagement durch verifizierte Angebote der EU einen Beitrag zur europäischen digitalen Souveränität leisten und die Machtposition von Google \& Co. einschränken können, da sie am ehesten in der Lage sind, die Merkmale Datenhoheit der Nutzer, Datenhaltung in Europa, Datenschutzkonformität und Vertrauenswürdigkeit durch Transparenz und Vertrauenswürdigkeit der assoziierten Partner zu gewährleisten. Zusätzlich spricht für nationale Lösungen, dass zur Fortentwicklung des E-Government verifizierte Partner unerlässlich sind [16].

Eine Schlussfolgerung dürfte sein, dass die Einhegung der US-Plattformen durch Regulierungen eine weitgehende Einigung der EU-Staaten voraussetzt und zugleich die Vorbereitung auf harte Gegenmaßnahmen der Trump-Administration mit sich bringen wird. Diese Ängste sind abzuwägen mit der stärker werdenden Unzufriedenheit der europäischen Öffentlichkeit nach politischer Kontrolle und
Regulierung der US-Plattformen, die solchen Bestrebungen Rückenwind geben sollte [6].

\section{Innovationen}

Für Europa bleibt das Pfund Forschung und Entwicklung kombiniert mit der schnellen Umsetzung in Innovationen. Im Bereich privater Service und Commerce sind die Big Five, von Ausnahmen abgesehen, nicht mehr einzuholen und bislang nur begrenzt einzuhegen, auch wenn mit dem geplanten und heftig diskutierten Digital Services Act die bisherigen Haftungsregeln zulasten der ,,marktdominanten Plattformen“ in der EU geändert werden sollten. Die Kräfte sind vor allem auf den industriellen und mittelständischen Bereich - eh eine Stärke des alten Kontinents - sowie auf Start-ups zu konzentrieren.

Für die Öffentlichkeit weitgehend unbemerkt geschieht hier mit cloudgestützten Vernetzungsplattformen im industriellen Bereich schon einiges. Es sind Transaktionsplattformen, die als Marktplätze fungieren und primär für den digitalen Vertrieb von industriellen Produkten eingesetzt werden sowie Internet-of-Things(IoT)-Plattformen zur digitalen Vernetzung der überbetrieblichen Produktion. IoTPlattformen vernetzen unabhängige Akteure und versetzen sie in die Lage, Fertigungs- und Lieferketten zu organisieren und Innovationen gemeinsam zu entwickeln. Die beteiligten Betriebe können so Maschinen- und Anlagedaten teilen und neue Geschäftsmodelle wie vorausschauende Wartungsprozesse etablieren [17]. Beispiele für IoT-Vernetzungsplattformen sind Air Supply, die kollaborative Prozesse entlang der Lieferkette der Luftfahrtindustrie erlaubt, oder Rail Supply, eine Plattform der Bahnindustrie, die die Zusammenarbeit von Bahnbetreiber, Hersteller und Zulieferer optimieren soll [18].

Trotz dieser Ansätze wird der ökonomische Wert von Daten und die Etablierung ganzer darauf aufbauender Ökosysteme von vielen mittelständischen Unternehmen noch wenig erkannt. Um nicht zuletzt dies zu ändern, haben im Juni 2020 die deutsche und französische Regierung das Großprojekt GAIA-X verabschiedet, das den Aufbau eines europäischen, vernetzten und offenen ,Datenökosystems“ zum Ziel hat. Genannt werden Kriterien wie IT-Sicherheit, Datenschutz, Offenheit und Transparenz, Authentizität und Vertrauen. Es sollen bislang dezentrale Infrastrukturdienste, beispielsweise IoT-Vernetzungsplattformen, zu einem nutzerfreundlichen System vernetzt werden, an dem auch außereuropäische Staaten teilnehmen können, sofern sie die Datensouveränität akzeptieren. Auf diese Weise sollen sich Unternehmen jeder Größe von Industrieunternehmen über mittelständische Unternehmen bis zu Start-ups verknüpfen [19].

GAIA-X ist eine Ansage an Cloud-Dienste wie Amazon, Google, Microsoft und Alibaba und ein Plädoyer für 
eine europäische Datensouveränität. Auf die Abhängigkeit besonders von US-Oligopolen und auf die daraus entstehende Gefahr für die EU, auf Dauer das liberale Wirtschaftsund Gesellschaftsmodell aufrecht erhalten zu können, wurde endlich von der deutschen und französischen Politik reagiert. Dieses Risiko wird in der GAIA-X-Präsentation explizit benannt, genauso wie die globalen Handelskonflikte und Spaltungen, die eine digitale Handlungsfähigkeit erforderlich machen.

Große deutsche Firmen wie Siemens und die Telekom sind dabei. Der Bundeswirtschaftsminister bezeichnet das Projekt als „Moonshot der Digitalpolitik“, als „,vielleicht wichtigstes digitales Bestreben einer Generation“" und hebt es auf eine Ebene mit dem „Airbus-Projekt", Metaphern und Ankündigungen, die wohl eher für die Galerie gedacht sind. Genauso wenig aber sollte man es als $\mathrm{zu}$ teuer, $\mathrm{zu}$ spät und angesichts des riesigen technischen Vorsprungs der Wettbewerber als unsinnig bezeichnen. Eher ist es die letzte Chance, europäische Souveränität in der digitalen Transformation zurückzugewinnen.

\section{Ideen und Diskussionen}

Daten im Verständnis der Wirtschaftsnobelpreisträgerin Elinor Ostrom als Allmendegüter einzustufen, also die Bewirtschaftung und Regelsetzung von Daten durch Gemeinschaften $\mathrm{zu}$ organisieren, ist einer von zahlreichen Vorschlägen, die diskutiert werden [20]. In eine ähnliche Richtung geht die Empfehlung, Daten als öffentliche Güter $\mathrm{zu}$ behandeln und dafür Datengenossenschaften zu gründen. Die Durchsetzung wird ähnlich schwierig sein wie die Zerschlagung der US-Oligopole oder das Verbot, mehrere Dienste anbieten zu dürfen. Durchsetzungsfähiger könnte die Idee sein, den in Deutschland verbreiteten Regulierungsansatz, Schutzräume auszuweiten, der heute u.a. bei der Datenschutzgrundverordnung (DSGVO) und bei der Preisbindung im Buchhandel Pate stand.

Im Rahmen der Forschungsförderung konkurrieren in Deutschland zwei Ansätze. Während die Max-Planck-Institute eher eine Kopie der amerikanischen DARPA-Agentur mit Sprunginnovationen favorisieren, empfehlen die Fraunhofer-Institute, den Schwerpunkt auf die Förderung und Kooperation von Mittelstand, Wissenschaftssystem und Startups zu legen.

Bei all diesen Diskussionen tritt zuweilen das kulturelle Narrativ in den Hintergrund. Schulen, Universitäten, Bibliotheken, Museen, öffentlich-rechtlicher Rundfunk u.a. sind Bausteine der Daseinsvorsorge und Kern des europäischen Gesellschaftsmodells. Google, Facebook, YouTube, Netflix \& Co. sind mit ihren Plattformen dabei, Spinnennetzen gleich die Kontrolle über den digitalen öffentlichen Raum und damit über das europäische Gesellschaftsmodell zu übernehmen [21]; deshalb die Empfehlung, das europäi- sche kulturelle Wertesystem als „digitale Wissensallmende“ neu zu organisieren.

Statt zuzusehen, wie auch europäische Kultur- und Medienproduzenten ihre Arbeiten auf YouTube oder Facebook stellen und damit diesen Daten und Profite liefern, sind europäische, beispielsweise genossenschaftliche Digitalplattformen, Stiftungen oder Public-Private-PartnershipKonstruktionen zu fördern. Dazu gibt es Diskussionen bei der ARD, beim ZDF, der European Broadcasting Union (EBU), die sowohl Konzepte wie Wikipedia, Open Access, Citizen Science bzw. Open Educational Resources (OER) berücksichtigen, als auch bei Überlegungen ansetzen, die europäische Medien-, Netz- und Digitalpolitik als Einheit zu betrachten.

\section{Ein europäisches Narrativ}

Ein europäisches Narrativ der digitalen Transformation ist möglich und nötig. Einige Beispiele, die den Digitalisierungspfad der EU positiv beeinflussen können, wurden erwähnt. Ein überzeugendes Narrativ beginnt damit, die verlorene digitale Regulierungshoheit wiederzugewinnen. Sie könnte den Bürgern das Vertrauen geben, dass die dominierenden US-Plattformen mit ihren Ansprüchen der Aushorchung, Kontrolle und Steuerung einzuhegen sind. Auch ihre ökonomische Dominanz, nicht zuletzt resultierend aus Steuerverkürzungen, ist zu beenden.

$\mathrm{Zu}$ einem europäisches Narrativ gehört, sich nicht die Innovationshoheit in der digitalen Forschung wie bei der praktischen Umsetzung in den Bereichen aus der Hand nehmen zu lassen, wo besonders Deutschland über Jahrzehnte weltmarktführend war. Bei der Umsetzung wird die EU ihr Augenmerk folglich vor allem auf die digitale Transformation der Groß- und mittelständischen Industrie legen. Die Bürger wollen sich auch auf eine produktive Balance zwischen demokratischen Ansprüchen, technisch-ökonomischem Fortschritt und Berücksichtigung von Klimafragen verlassen können, in toto also, dass ein nachhaltiger Digitalisierungspfad verfolgt wird. Und schließlich sollte das Überleben der europäischen Kultur in Zeiten von YouTube, Streamingdiensten, Facebook und Google kein Restposten sein, sondern ein prioritäres Ziel mit finanzieller Förderung werden.

\section{Literatur}

1. Kuhn T (1989) Die Struktur wissenschaftlicher Revolutionen (Org 1962)

2. Fleck L (1935) Entstehung und Entwicklung einer wissenschaftlichen Tatsache. Einführung in die Lehre von Denkstil und Denkkollektiv

3. Polanyi K (2015) The Great Transformation. Suhrkamp, Berlin (Org. 1944) 
4. McAfee A, Brynjolfsson E (2018) Machine, Platform, Crowd: Wie wir das Beste aus unserer digitalen Zukunft machen. Plassen, Plassen-Verlag Kulmbach

5. Rudolph M (2020) Der Weltgeist als Lachs. Geschichtsphilosophische Implikationen des chinesischen Aufstiegs. Merkur 74(849):2

6. netzpolitik.org (3.5.29017), https:// netzpolitik.org/2017/steigendeabhoerzahlen-bei nsa-ueberwachung/

7. Dolata U (2020) Plattform-Regulierung. Koordination von Märkten und Kuratierung von Sozialität im Internet. Berlin J Soziol. https:// doi.org/10.1007/s11609-020-00403-9

8. Hoffmann-Riem W (2020) Digitale Disruption - Herausforderungen für Recht und Rechtswissenschaft. In: Eifert M (Hrsg) Digitale Disruption und Recht. Nomos, Baden-Baden

9. Lessig L (1999) CODE and other laws of cyberspace. Basic Books, New York

10. Zuboff S (2018) Das Zeitalter des Überwachungskapitalismus. Campus, Frankfurt/M.

11. Pfeiffer S (2016) Diskurs und Strategie. FifF-Kommunikation 2016(4):12-17

12. Schwab, Klaus (2019): Rede auf Davos Forum 2019, abgedruckt in: FAZ 22.1.19, Carsten Knop: Digitale Erneuerer statt Sklaven der Algorithmen.

13. Mingels, Guido (2020): Und es hat Zoom gemacht. In: Der Spiegel, Nr. 23/2020
14. Reckwitz, Andreas (2020): „Verblendet vom Augenblick“, In: DIE ZEIT vom 10.06.2020, S. 45

15. Simon J (2016) Values in Design. In: Heesen J (Hrsg) Handbuch Medien- und Informationsethik. J.B. Metzler, Stuttgart

16. Brecht ML (2020) Neue Monopole im Kontext der digitalen Transformation - Risiken und Strategien am Beispiel digitaler Identitäten und der Identitätsplattform 〈Verimi, Bachelorarbeit des FB Informatik. Universität Hamburg, Hamburg

17. BMWI (2019a) Die volkswirtschaftliche Bedeutung von digitalen B2B-Plattformen im Verarbeitende Gewerbe. https://www.de. digital/DIGITAL/Redaktion/DE/Digital-Gipfel/Download/2019/ digitale-b2b-plattformen-im-verarbeitenden-gewerbe.pdf? blob=publicationFile \&v=3. Zugegriffen: 3.6.2020

18. Stiel, Hadi (2020): Wie Plattformen die Ökonomie verändern, In. FAZ 5. März 2020

19. BMWI (2019): GAIA-X Eine vernetzte Dateninfrastruktur als Wiege eines vitalen, europäischen Ökosystems, Oktober 2019

20. Schneider I (2019) Regulierungsansätze in der Datenökonomie, Bundeszentrale für Politische Bildung 2019. https://www.bpb.de/ apuz/292347/regulierungsansaetze-in-der-datenoekonomie. Zugegriffen: 4.6.2020

21. Rolf A (2018) Weltmacht Vereinigte Daten. Die Digitalisierung und Big Data verstehen. metropolis, Marburg 